\title{
Common pathways to NSSI and suicide ideation: The roles of rumination and self-compassion
}

\section{Penelope Hasking, Mark E Boyes, Amy Finlay-Jones, Peter M. McEvoy \& Clare} S. Rees

To cite this article: Penelope Hasking, Mark E Boyes, Amy Finlay-Jones, Peter M. McEvoy \& Clare S. Rees (2018): Common pathways to NSSI and suicide ideation: The roles of rumination and self-compassion, Archives of Suicide Research, DOI: 10.1080/13811118.2018.1468836

To link to this article: https://doi.org/10.1080/13811118.2018.1468836

Accepted author version posted online: 23

May 2018.

Submit your article to this journal $[\pi$

Џ Article views: 26

View Crossmark data \lceil 


\title{
Common pathways to NSSI and suicide ideation:
}

\section{The roles of rumination and self-compassion}

\author{
Penelope Hasking $^{1 *}$, Mark E Boyes ${ }^{1}$, Amy Finlay-Jones ${ }^{1,2}$, \\ Peter M. McEvoy ${ }^{1,3}$, Clare S. Rees ${ }^{1}$
}

${ }^{1}$ School of Psychology and Speech Pathology, Curtin University, Perth, Australia

${ }^{2}$ Telethon Kids Institute, Perth, Australia

${ }^{3}$ Centre for Clinical Interventions, Perth, Australia

*Corresponding author: Penelope Hasking, School of Psychology and Speech Pathology,

Curtin, University, Perth, Australia. Ph: + 6189266 3437. E:

Penelope.Hasking@curtin.edu.au

Acknowledgements: This study was supported, in part, by a grant awarded to Peter McEvoy, Penelope Hasking, and Clare Rees by the Research and Development Committee of the School of Psychology and Speech Pathology, Faculty of Health Sciences, Curtin University (SRAF-2014-37).

Word count: 4746

Running head: Self-compassion, rumination, NSSI and suicide 


\title{
Common pathways to NSSI and suicide ideation:
}

\section{The roles of rumination and self-compassion}

\begin{abstract}
Objectives: We investigated whether rumination and self-compassion moderate and/or mediate the relationships between negative affect and both non-suicidal self-injury (NSSI) and suicide ideation. Methods: Undergraduate university students $(n=415)$ completed wellvalidated measures of negative affect, rumination, self-compassion, NSSI, and suicide ideation. Results: Neither rumination nor self-compassion moderated associations between negative affect and NSSI and suicide ideation. However, both rumination and selfcompassion mediated associations between negative affect and life-time history of NSSI and suicide ideation. Self-compassion additionally mediated the association between negative affect and both 12 month NSSI and suicide ideation. Conclusion: The salience of selfcompassion, particularly in predicting recent NSSI and suicide ideation, offers promise for early intervention initiatives focusing on less judgmental or self-critical means of selfrelation.
\end{abstract}

Keywords: NSSI, suicide ideation, self-compassion, rumination 
Up to one in five young adults report a history of non-suicidal self-injury (NSSI), the deliberate destruction of body tissue without conscious suicidal intent (Nock, 2009; Swannell, Martin, Page, Hasking, \& St John, 2014). NSSI is typically used to relieve intense negative affect, and is associated with a number of adverse outcomes including poor academic performance, and comorbid psychopathology (Bentley, et al., 2015; Bentley, Nock \& Barlow, 2015; Hamza \& Willoughby, 2016; Kiekens et al., 2016). Despite being engaged with no conscious suicidal intent, NSSI is strongly associated with suicidal thoughts and behaviour (Ribeiro et al., 2016). While NSSI may be a precursor to subsequent suicide ideation (Whitlock et al., 2013), a significant proportion of young people experience both NSSI and suicide ideation within the same year (Glenn et al., 2017; Hamza \& Willoughby, 2016). There is growing evidence that different cognitive-emotional factors underlie NSSI and suicidal thoughts (Fox et al., 2015; Franklin et al., 2017), suggesting that better understanding of common and differential pathways to NSSI and suicide ideation may assist in targeted prevention and early intervention initiatives to reduce both suicidal and nonsuicidal behaviours. Of note, while significant efforts have been made to understand factors which might increase risk, more work is needed to identify factors that might reduce risk of suicidal and non-suicidal behaviours used to alleviate negative affect.

\section{Negative affect, NSSI, and suicide ideation}

Although NSSI can serve a number of functions for an individual, the most frequently cited motive for self-injury is to regulate intense or unwanted affect. People reporting heightened negative emotions are more likely to self-injure (Nicolai, Wielgus, \& Mezulis, 2016; Nock \& Prinstein, 2005), and people who self-injure report greater reductions in negative affect following the application of a painful stimulus (Bresin \& Gordon, 2013). Daily diary studies also show heighted negativity on days when people self-inure, and reductions in negative affect following NSSI, supporting the affect regulation function 
(Muehlenkamp et al., 2009; Turner, et al., 2016). Similarly, sensitivity to negative affect, and the intensity with which negative affect is experienced, are temporally related to suicide ideation (Selby, Yen, \& Spirito, 2013). While it seems clear that negative affect is related to both NSSI and suicidal ideation, the pathways by which negative affect can lead to these outcomes are less clear. Specifically, while NSSI and suicide ideation could be two outcomes of a common pathway from negative affect it is just as likely that negative affect exerts an effect on different underlying mechanisms in the pathways to NSSI and ideation. In this paper we explore rumination and self-compassion as two such pathways.

\section{Rumination}

Rumination is a transdiagnostic cognitive process consisting of repetitive attempts to analyse problems and feelings of distress, without taking the necessary action to make positive changes (Harvey, Watkins, Mansell, \& Shafran, 2004; McEvoy, Mahoney \& Moulds, 2010; McEvoy, Watson, Watkins \& Nathan, 2013; Nolen-Hoeksema, 1991). Rumination is associated with increases in negative thoughts and images, and reduces the likelihood that individuals will engage in more adaptive emotion regulation strategies (McEvoy, Hayes, Hasking \& Rees, 2017; Watkins, 2015). In describing Emotional Cascade Theory, Selby and Joiner (2009) explain how rumination can increase use of emotionregulatory behaviours such as NSSI. They argue that rumination on negative stimuli can exacerbate negative affect, intensify the cycle, and result in emotional cascades of increasingly intense emotion (Selby \& Joiner, 2009). Given the intensity of the emotion, simple distraction techniques (e.g., going for a walk) are often insufficient to interrupt the cascade. However, NSSI can effectively disrupt the cascade by providing a physical diversion from the distressing emotional state. The resulting reduction in arousal and negative affect then maintains use of NSSI in distressing situations. 
Self-report, experimental, and ecological momentary assessment studies offer support for Emotional Cascade Theory, evidencing strong links between escalating rumination and NSSI (Arbuthnott, Lewis, \& Bailey, 2015; Bresin \& Verona, 2016; Nicolai, et al., 2016; Selby, Franklin, Carson-Wong, \& Rizvi, 2013). Similarly, there is a strong relationship between rumination and suicide ideation (Morrison \& O'Connor, 2008; Rogers and Joiner, 2017). Rumination demonstrates cross-sectional and prospective relationships with suicide ideation and suicide attempts (Morrison \& O'Connor, 2008), and mediates the relationship between cognitive vulnerability and suicide ideation (Miranda, et al., 2013; Smith, Alloy \& Abramson, 2006; Surrence, Miranda, Marroquín, \& Chan, 2009). Consequently, rumination may be a common cognitive vulnerability for both NSSI and suicide attempts.

\section{Self-compassion}

While rumination can exacerbate negative affect, less work has explored factors that might protect against NSSI and suicidal ideation when feeling negative emotion. One potential factor is self-compassion, an adaptive form of self-relation characterised by the tendency to respond to difficult experiences in a balanced and self-nurturing way, while acknowledging that suffering is part of the common human experience (Neff, 2003). Previous work has found that self-compassion promotes adaptive self-regulation in the face of difficult emotions (Arch et al, 2014; Breines et al. 2014), and is inversely linked with maladaptive cognitions such as rumination (Finlay-Jones, 2017; Raes, 2010). Conversely, low self-compassion - characterised by high levels of self-judgement, over-identification with difficult emotions (i.e., "I feel bad therefore I am bad"), and feelings of isolation during times of suffering - may increase the likelihood that one may self-injure (Xavier, PintoGouveia, \& Cunha, 2016). Supporting this, self-compassion is negatively associated with NSSI thoughts and actions, and moderates the relationship between depression and NSSI in adolescents (Jiang, You, Ren, et al., 2017; Jiang, You, Zheng, \& Lin, 2017). Previous work 
has also found that self-compassion moderates the relationship between negative affect and symptoms of depression and anxiety (Trompetter et al., 2017). However, it is also plausible that self-compassion mediates the link between negative affect and both NSSI and suicide ideation, such that high negative affect undermines self-compassion and leads to greater NSSI and suicide ideation.

\section{The current study}

In the current study we aimed to determine whether rumination and self-compassion moderate the relationship between negative affect and both NSSI and suicide ideation separately, such that rumination increases risk of NSSI and suicide ideation in the context of negative affect, while self-compassion reduces this risk. We also assessed whether negative affect exerts an indirect effect on these outcomes, working through rumination and selfcompassion. Finally, given that factors underlying any history of NSSI or suicide ideation may differ from pathways which maintain behaviour (Taliaferro \& Muehlenkamp, 2015), we assessed these relationships both in predicting odds of any history of NSSI and/or suicide ideation, and among people reporting 12-month NSSI and/or ideation.

\section{Method}

\section{Participants \& Procedure}

The sample comprised 415 undergraduate psychology students enrolled in different units across the first three years of their undergraduate degree (Mean age $=20.99, S D=5.33$ ). The majority were female $(n=317,76.8 \%)$. Most were studying full time $(93.2 \%)$ and, as is typical in Australia, living at home with parents/family $(n=286,69.1 \%)$. The study was promoted to students via an electronic notice board advertising studies in which students could participate for course credit. The study was advertised as an examination of different emotion regulation strategies and psychological distress. The information sheet explicitly called for both participants who self-injured and those who did not. Interested students were 
directed to an online questionnaire where they completed the following measures as part of a larger study. All participants were provided information about the aims of the study, participation requirements, confidentiality of responses, and compliance with secure data handling procedures. Upon completion, participants were provided information sheets about NSSI and mental health, as well as a list of local mental health resources. The study was approved by the (blinded for review) Human Research Ethics Committee.

\section{Measures}

Nonsuicidal self-injury. Self-injury was assessed with Section I of the Inventory of Statements about Self-Injury (Klonsky \& Olino, 2008), a self-report measure enquiring about engagement in 12 common forms of NSSI (e.g., cutting, burning, self-battery). The measure has sound test-retest reliability ( $r=.85$; Klonksy \& Olino, 2008). Participants were also asked how often they have engaged in NSSI in the last 12 months (once, twice, three times, four times, 5+ times). History of NSSI was coded as $0=$ no history, $1=$ lifetime history. Frequency of NSSI in the last year was used as an ordinal variable in analyses.

Suicide ideation. To assess suicide ideation, participants were asked "In the last 12 months have you thought about ending your life?" and "Have you ever thought about ending your life?" The dichotomous responses to these questions $(0=$ no, $1=$ yes $)$ were used in analyses.

Negative affect. Negative affect was assessed with the negative affect scale of the Positive and Negative Affect Schedule (PANAS; Watson, Clarke \& Tellegan, 1988). In the negative affect subscale, participants are presented with 10 negative emotions (e.g., ashamed, distressed) and asked to rate the extent to which they have experienced each emotion over the past week. Responses are summated to provide a total scale score. This scale evidences construct validity, reliability, and measurement invariance across demographic groups (Crawford \& Henry, 2004). In this sample, internal consistency was $\alpha=.86$. 
Rumination. Rumination was assessed with the 10-item Repetitive Thinking Questionnaire (RTQ; McEvoy, et al., 2010; McEvoy, Thibodeau, \& Asmundson, 2014). Participants responded to items assessing their tendency to think negatively when feeling distressed (e.g., "I think about the situation all the time"), on a 5-point Likert scale. Item responses are summated to produce a total score. The RTQ has demonstrated reliability and validity (McEvoy et al., 2010; 2014). Internal consistency was evident in the current sample, $\alpha=.93$.

Self-compassion. We assessed self-compassion with the short form of the SelfCompassion Scale (Raes, Pommier, Neff, \& van Gucht, 2011), a 12-item measure of how an individual responds to themselves when upset. Six subscale scores reflect: self-kindness (e.g. "I try to be understanding and patient towards those aspects of my personality I don't like"), self-judgment (e.g. "I'm disapproving and judgmental about my own flaws and inadequacies"), common humanity (e.g. "I try to see my failings as part of the human condition"), isolation (e.g. "When I'm feeling down, I tend to feel like most other people are probably happier than I am"”), mindfulness (e.g. "When something painful happens I try to take a balanced view of the situation"), and over-identification (e.g. "When I'm feeling down I tend to obsess and fixate on everything that's wrong"). For each subscale, item scores are summated. Participants indicate the degree to which they endorse each statement on a 5-point scale. The short form correlates almost perfectly with the longer version, although subscale scores tend to be less reliable (Raes et al., 2011). Consistent with initial validation of the shortened scale, in our sample internal consistency for the subscales ranged from $\alpha=.53-.83$.

\section{Data analysis}

No variable had more than $10 \%$ missing data (range $1.4 \%$ to $9.2 \%$ ) and data were missing completely at random, $\chi^{2}(157)=73.08, p=1.00$. Expectation maximisation was used to impute missing data. As outlined above, the relationship between negative affect and either 
NSSI or suicide ideation could work indirectly through rumination and self-compassion, or the relationships could be moderated by these variables. For the sake of parsimony in model testing, we first conducted a series of moderated logistic regressions to predict: lifetime history of NSSI, lifetime suicide ideation, and 12 month suicide ideation. We also conducted a multinomial regression to predict frequency of NSSI in the last year. No significant interactions terms were observed in any of the analyses (all $p \mathrm{~s}>.05$ ). As such, we tested direct and indirect effects of NA, rumination, and self-compassion on the outcomes, but did not test moderation.

Two models were tested. In the first, lifetime NSSI (no/yes) and lifetime suicide ideation (yes/no) were entered as the two criterion variables. In the second, we restricted the sample to participants who had self-injured or reported suicide ideation in the last 12 months, and used frequency of NSSI in the last 12 months, and 12-month suicide ideation (no/yes), as the two criterion variables. In both models, direct effects of negative affect, rumination, and selfcompassion on the criterion variables were assessed, as were indirect effects of negative affect working though rumination and self-compassion. Given some subscale scores had poor internal consistency, we entered self-compassion as a latent variable, indicated by subscale scores; all other variables were observed variables. Self-reported history of any mental illness was statistically controlled in all analyses (i.e., "Have you ever been diagnosed with a mental illness"). All models were tested using the WLSMV estimator in MPlus v7.4 (Muthén \& Muthén, 2012). Model fit was determined by: $\chi^{2} / \mathrm{df} \leq 3$, Comparative Fit Index $(\mathrm{CFI})>.95$, Tucker Lewis Index $(\mathrm{TLI})>.95$, Root Mean Error of Approximation $($ RMSEA) $<.05$, and Weighted Root Mean Square Residual (WRMR) < 1.0 (Hu \& Bentler, 1999) 


\section{Results}

\section{Sample characteristics}

Of the sample, $33.1 \%(n=135)$ reported a history of NSSI, with 70.1\% $(n=87)$ selfonce or twice in the last year. Half $(50.4 \%, n=202)$ reported ever experiencing suicidal ideation, and $22.4 \%(n=90)$ reported ideation in the last 12 months. Of the sample, $24.8 \%$ reported a lifetime history of both NSSI and suicide ideation; $12.53 \%$ reported both NSSI and suicide ideation in the last 12 months.

Neither history of NSSI, nor frequency in the last twelve months, were related to gender or age (all $p \mathrm{~s}>.05)$. One quarter of the sample $(24.6 \%)$ self-reported they had been diagnosed with a mental illness at some point in their life, most commonly depressive (74.7\%) and anxiety (44.19\%) disorders. History, but not frequency, of NSSI was associated with a mental illness diagnosis, $\chi^{2}(1)=30.74, p<.001$. Similarly, neither past ideation, nor recent ideation, were associated with age or gender of participants (all $p \mathrm{~s}>.05$ ). Both past ideation, $\chi^{2}(1)=52.74, p<.001$, and recent ideation, $\chi^{2}(1)=34.442, p<.001$, were associated with mental illness. The majority of variables were correlated in the expected direction (Table 1).

\section{Lifetime NSSI and ideation}

The model predicting lifetime NSSI and lifetime suicide ideation fit the data well, $\chi^{2}(15)$ $=23.58, p=.07, \chi^{2} / \mathrm{df}=1.57, \mathrm{CFI}=.99, \mathrm{TLI}=.97, \mathrm{RMSEA}=.04, \mathrm{WRMR}=.47$. Neither NSSI nor suicide ideation were directly related to NA, but rumination and self-compassion were (Figure 1). There were significant indirect effects of NA on both NSSI and suicidal ideation, working through both rumination and self-compassion (Table 2).

\section{2 month NSSI frequency and 12-month suicide ideation}

The model predicting 12-month NSSI and suicide ideation was an adequate fit to the data, $\chi^{2}(35)=58.07, p=.01, \chi^{2} / \mathrm{df}=1.66, \mathrm{CFI}=.94, \mathrm{TLI}=.90, \mathrm{RMSEA}=.06, \mathrm{WRMR}=$ 
.78. Again, neither NSSI frequency nor suicidal ideation were directly related to NA, nor was rumination. Indirect paths through self-compassion were observed for both outcomes (Table 2).

\section{Discussion}

The aim of the current study was to explore whether rumination and self-compassion moderate links between negative affect, NSSI and suicide ideation, or if negative affect exerts an indirect effect on NSSI and suicide ideation, working through rumination and selfcompassion. Surprisingly, rumination and self-compassion did not moderate the affect-NSSI relationship. Instead, negative affect was seen to exert an indirect effect on lifetime history of both NSSI and suicide ideation, working though both rumination and self-compassion. Specifically, high negative affect related to more rumination and less self-compassion, which in turn increased odds of NSSI and suicide ideation.

Our analyses returned different results when predicting recent NSSI and suicide ideation; here lack of self-compassion was observed to underlie the relationship between negative affect and NSSI, whereas rumination was not related. Consistent with previous research (e.g. Jiang et al, 2017; Xavier et al., 2016), this suggests that individuals who are prone to responding to difficult emotional experiences in an over-identified, self-isolating, and selfjudgemental manner may be more likely to engage in NSSI or suicide ideation. On the other hand, these outcomes are less likely amongst individuals who are able to respond to negative emotions in a balanced and self-nurturing way, and see the common humanity in their hardships and difficult experiences. These findings suggest that while a general tendency to ruminate is related to any history of suicidal thoughts and behaviours, ongoing suicidal thoughts and behaviour relate more strongly to a lack of self-compassion. 


\section{Treatment implications}

The current study was cross-sectional and included a non-clinical sample, but if the observed pattern of relationships is replicated within prospective or experimental studies they would have important implications for interventions that target both rumination and selfcompassion. The degree to which negative affect leads to rumination may determine risk of NSSI or suicide ideation, making rumination a particularly salient target for prevention as well as intervention. Some recent approaches have been developed that attempt to directly target rumination by helping individuals to step-out of habitual patterns of rumination. Mindfulness-Based Cognitive Therapy (MBCT; Segal, Williams \& Teasdale, 2002) is a group-based intervention that combines elements of cognitive therapy (including efforts to reduce rumination) with mindfulness practices, with demonstrated efficacy in reducing depression vulnerability. Rumination-Focused Cognitive Behavioural Therapy (Watkins et al. 2007) builds on conventional cognitive-behavioural therapy (CBT) by using functional analysis to explore how, where, and when rumination occurs and its function with the aim to systematically reduce or replace it.

Although tentative, our findings suggest self-compassion might be an important mechanism underlying the relationship between negative affect and both NSSI and suicide ideation. Because self-compassion spans a dimension from actively self-critical to actively self-soothing, this can be interpreted in two ways. First, in individuals with low trait selfcompassion, negative affect could trigger overidentification with the negative emotional experience, leading to self-criticism, self-isolation, and a tendency to engage in NSSI or suicide ideation. Second, amongst individuals with high trait self-compassion, the experience of negative affect might prompt a mindful response to the difficult experience, acting as a reminder that negative emotions are shared by all humans, and triggering an actively selfsoothing response. In this context, self-compassion may act as an emotion regulation strategy 
that removes the need for alternatives (such as NSSI) to reduce feelings of distress. Further work is required to confirm these potential mechanisms.

A number of interventions are now available to support the cultivation of selfcompassion, including the Mindful Self-Compassion program (Neff \& Germer, 2013), the Compassion Cultivation Training Program (Jazaieri et al., in press), the Mindfulness-Based Compassionate Living program (van den Brink \& Koster, 2015), and Compassion-Focused Therapy (CFT; Gilbert, 2009; 2014). These interventions - particularly CFT - have been trialled with individuals experiencing clinical and subclinical symptoms of psychopathology, including depression, anxiety, psychosis, and eating disorders. To our knowledge, no previous work has examined the efficacy of self-compassion-based interventions for reducing NSSI or suicide ideation in at-risk individuals.

\section{Limitations and research implications}

As noted above, our findings require replication in larger, more representative, gender balanced, and clinical samples. The fact that students self-selected into our study naturally raises the possibility of self-selection bias, although our sample demographics, and NSSI distributions, are similar to other studies using the same methodology (e.g. Hasking \& Rose, 2016). Our finding that rumination was not related to recent NSSI or suicide ideation may be an artefact of our design rather than an indication that rumination is not implicated in recent NSSI or ideation. Specifically, we assessed repetitive negative thinking as a broad construct. Prior work has suggested different facets of rumination (e.g., brooding, reflection; PolancoRoman, et al.,2015) might be differentially related to suicidal and non-suicidal outcomes, suggesting a more nuanced assessment of rumination may be warranted. We assessed negative affect over the last week, but assessed any prior NSSI and suicide ideation. Due to the cross-sectional nature of the data we cannot make temporal inferences, although correlations between trait and state affect (Komulainen et al., 2014; Miller, Vachon, \& 
Lynam, 2010) suggest assessing trait negative affect is likely to have given similar findings. However future work should assess constructs across similar timeframes. Further, Emotional Cascade Theory proposes a dynamic interplay between rumination and affect, resulting in an escalation of the emotional experience. Momentary assessment studies, rather than assessment of trait rumination, are required to understand the complex interplay between affect and rumination, and to determine whether rumination moderates the relationship between negative affect and outcomes such as NSSI and suicide ideation. Finally, any treatment programs designed to reduce rumination or enhance self-compassion must necessarily be evaluated for their ability to reduce NSSI and suicidal ideation. Further, the proposed mechanisms of any treatment effect must be established before confirming whether changes in rumination and self-compassion are fundamental to producing positive change in suicidal and non-suicidal behaviour.

\section{Conclusion}

The above limitations notwithstanding, our findings suggest that negative affect exerts an indirect effect on NSSI and suicide ideation, working through rumination and selfcompassion. The salience of self-compassion, particularly in predicting recent NSSI and suicide ideation, offers promise for early intervention initiatives focusing on less judgmental or self-critical means of self-relation. Further work exploring whether fostering selfcompassion reduces the frequency and severity of NSSI and/or suicide ideation is warranted. 


\section{References}

Arbuthnott, A.E., Lewis, S.P., \& Bailey, H.N. (2015). Rumination and emotions in nonsuicidal self-injury and eating disorder behaviours: A preliminary test of the emotional cascade model. Journal of Clinical Psychology, 71, 62-71

Arch, J.J., Brown, K.W., Dean, D.J., Landy, L.N., Brown, K.D., \& Laudenslager, M.L. (2014) Self-compassion training modulates alpha-amylase, heart rate variability, and subjective responses to social evaluative threat in women. Psychoneuroendocrinology, 42, 49-58.

Bentley, K.H., Cassiello-Robbins, C.F., Vittorio, L., Sauer-Zavala, S., \& Barlow, D.H. (2015). The association between nonsuicidal self-injury and the emotional disorders: A meta-analytic review. Clinical Psychology Review, 37, 72-88.

Bentley, K.H., Nock, M.K., \& Barlow, D.H. (2015). The four-function model of nonsuicidal self-injury. Clinical Psychological Science, 2, 638-656.

Breines, J.G., Thoma, M.V., Gianferante, D., Hanlin, L., Chen, X., \& Rohleder, N. (2014). Self-compassion as a predictor of interleukin-6 response to acute psychosocial stress. Brain Behave Immunology, 37, 109-114.

Bresin, K., \& Gordon, K.H. (2013). Changes in negative affect following pain (vs nonpainful) stimulation in individuals with and without a history of nonsuicidal selfinjury. Personality Disorders, 4, 62-66.

Bresin, K., \& Verona, E. (2016). Pain, affect and rumination: An experimental test of the Emotional Cascade Theory in two undergraduate samples. Journal of Experimental Psychopathology, 7, 205-224.

Crawford, J.R., \& Henry, J.D. (2004). The positive and negative affect schedule (PANAS): Construct validity, measurement properties and normative data in a large non-clinical sample. British Journal of Clinical Psychology, 43, 245-265. 
Finlay-Jones, A.L. (2017). The relevance of self-compassion as an intervention target in mood and anxiety disorders: A narrative review based on an emotion regulation framework. Clinical Psychologist, 21, 90-103.

Fox, K.R., Franklin, J.C., Ribeiro, J.D., Kleiman, E.M., Bentley, K.H., \& Nock, M.K. (2015). Meta-analysis of risk factors for nonsuicidal self-injury. Clinical Psychology Review, 42, $156-167$.

Franklin, J.C., Ribeiro, J.D., Fox, K.R., Bentley, K.H., Kleiman, E.M., Huang, X., Musacchio, K.M., Jaroszewski, A.C., Change, B.P., \& Nock, M.K. (2017). Risk factors for suicidal thoughts and behaviours: A meta-analysis of 50 years of research. Psychological Bulletin, 143, 187-232.

Gilbert, P. (2014). The origins and nature of compassion focused therapy. British Journal of Clinical Psychology, 53, 6-41.

Gilbert, P. (2009). Introducing compassion focused therapy. Advances in Psychiatric Treatment, 15, 199-208.

Glenn, C.R., Lanzillo, E.C., Esposito, E.C., Santee, A.C., Nock, M.K., \& Auerbach, R.P. (2017). Examining the course of suicidal and nonsuicidal self-injurious thoughts and behaviours in outpatient and inpatient adolescents. Journal of Abnormal Child Psychology, 45, 971-983

Hamza, C.A., \& Willoughby, T. (2016). Nonsuicidal self-injury and suicidal risk among emerging adults. Journal of Adolescent Health, 59, 411-415.

Harvey, A., Watkins, E., Mansell, W., \& Shafran, R. (2004). Cognitive behavioural processes across psychological disorders: A transdiagnostic approach to research and treatment. Oxford: Oxford University Press.

Hasking, P., \& Rose, A. (2016). A preliminary application of social cognitive theory to nonsuicidal self-injury. Journal of Youth and Adolescence, 45, 1560-1574. 
Hu, L., \& Bentler, P.M. (1999). Cutoff criteria for fit indexes in covariance structure analysis: Conventional criteria versus new alternatives. Structural Equation Modeling, 6, 1-55.

Jazaieri, H., McGonigal, K., Lee, I.A., Jinpa, T., Doty, J.R., Gross, J.J., \& Goldin, P.R. (in press). Altering the trajextory of affect and affect regulation" The imapct of compassion training. Mindfulness.

Jiang, Y., You, J., Ren, Y., Sun, R., Liao, S., Zhu, J., \& Ma, N. (2017). Brief report: A preliminary comparison of self-compassion between adolescents with nonsuicidal selfinjury thoughts and actions. Journal of Adolescence, 59, 124-128.

Jiang, Y., You, J., Zheng, X., \& Lin, M.-P. (2017). The qualities of attachment with signficiant others and self-compassion protect adolescents from non-suicidal self-injury. School Psychology Quarterly, 32(2), 143-155.

Kiekens, G., Claes, L., Demyttenaere, K., Auerbach, R., Green, J.G., Kessler, R.C., Mortier, P., Nock, M.K., \& Bruffaerts, R. (2016). Lifetime and 12-month nonsuicidal self-injury and academic performance in college freshman. Suicide and Life-Threatening Behaviour, 46, 563-576.

Klonsky, E. D., \& Olino, T. M. (2008). Identifying clinically distinct subgroups of selfinjurers among young adults: A latent class analysis. Journal of Consulting \& Clinical Psychology, 76, 22.

Komulainen, E., Meskanen, K., Lipsanen, J., Lahti, J.M., Jylhä, P., Melartin, T., Wichers, M., Isometsä, E., \& Ekelund, J. (2014). The effect of personality on daily life emotional processes. PLOS ONE, 9(10).

McEvoy, P. M., Erceg-Hurn, D. M., Saulsman, L. M., \& Thibodeau, M. A. (2015). Imagery enhancements increase the effectiveness of cognitive behavioural group therapy for social anxiety disorder: a benchmarking study. Behaviour Research and Therapy, 65, 42-51. 
McEvoy, P. M., Hayes, S., Hasking, P. S., \& Rees, C. S. (2017). Thoughts, images, and appraisals associated with acting and not acting on the urge to self-injure. Journal of Behavior Therapy and Experimental Psychiatry, 57, 163-171.

McEvoy, P. M., Mahoney, A. E. J., \& Moulds, M. L. (2010). Are worry, rumination, and post-event processing one and the same? Development of the Repetitive Thinking Questionnaire, Journal of Anxiety Disorders, 24, 509-515.

McEvoy, P. M., Watson, H., Watkins, E., \& Nathan, P. (2013). The relationship between worry, rumination, and comorbidity: Evidence for repetitive negative thinking as a transdiagnostic construct. Journal of Affective Disorders, 151, 313-320.

Miller, D.J., Vachon, D.D., \& Lynam, D.R. (2010). Neuroticism, negative affect, and negative instability: Establishing convergent and discriminant validity using ecological momentary assessment. Personality and Individual Differences, 47, 873-877.

Miranda, R., Valderrama, J., Tsypes, A., Gadol, E., \& Gallgher, M (2013). Cognitive inflexibility and suicidal ideation: mediating role of brooding and hopelessness. Psychiatry Research, 210, 174-181.

Morrison, R., \& O’Connor, R.C. (2008;). A systematic review of the relationship between rumination and suicidality. Suicide and Life-Threatening Behavior, 38, 523-538.

Muehlenkamp, J.J., Engel, S.G., Wadeson, A., Crosby, R.D., Wonderlich, S.A., Simonich, H., \& Mitchell, J.E. (2009). Emotional states preceding and following acts of non-suicidal self-injury in bulimia nervosa patients. Behaviour, Research and Therapy, 47, 83-87.

Muthén, L.K. and Muthén, B.O. (1998-2012). Mplus User’s Guide. Seventh Edition. Los Angeles, CA: Muthén \& Muthén.

Neff, K. (2003). Self-compassion: An alternative conceptualisation of a healthy attitude toward oneself. Self and Identity, 2, 85-101. 
Neff, K.D., \& Germer, C.K. (2013). A pilot study and randomized controlled trial of the mindful self-compassion program. Journal of Clinical Psychology, 69, 28-44.

Nicolai, K.A., Wielgus, M.D., \& Mezulis, A. (2016). Identifying risk for self-harm: Rumination and negative affectivity in the prospective prediction of nonsuicidal selfinjury. Suicide \& Life-Threatening Behavior, 46, 223-233.

Nock, M.K. (2009). Why do people hurt themselves? New insights into the nature and functions of self-injury. Current Directions in Psychological Science, 18(2), 78-83.

Nock, M.K. \& Prinstein, M.J. (2005). Contextual features and behavioural functions of selfmutilation among adolescents. Journal of Abnormal Psychology, 114, 140-146.

Nolen-Hoeksema, S. (1991). Responses to depression and their effects on the duration of depressive episodes. Journal of Abnormal Psychology, 100, 569-582.

Polanco-Roman, L., Jurska, J., Quiñones, V., \& Miranda, R. (2015). Brooding, reflection, and distraction: Relation to nonsuicidal self-injury versus suicide attempts. Archives of Suicide Research, 19, 350-365.

Raes, F. (2010). Rumination and worry as mediators of the relationship between selfcompassion and depression and anxiety. Personality and Individual Differences, 48, 757761.

Raes, F., Pommier, E., Neff, K. D., \& Van Gucht, D. (2011). Construction and factorial validation of a short form of the Self-Compassion Scale. Clinical Psychology and Psychotherapy, 18, 250-255.

Ribeiro, J.D., Franklin, J.C., Fox, K.R., Bentley, K.H., Kleiman, E.M., Chang, B.P., 7 Nock, M.K. (2016). Self-injurious thoughts and behaviours as risk factors for future suicide ideation, attempts, and death: A meta-analysis of longitudinal studies. Psychological Medicine, 46, 225-236. 
Rogers, M. L., \& Joiner, T. E. (2017). Rumination, suicidal ideation, and suicide attempts: A meta-analytic review. Review of General Psychology, 21, 132-142.

Segal, Z.V., Williams, J.M.G., \& Teasdale, J.D. (2002). Mindfulness-based cognitive therapy for depression: A new approach to preventing relapse. New York: Guildford Press.

Selby, E.A., Franklin, J., Carson-Wong, A., \& Rizvi, S.L. (2013). Emotional cascades and self-injury: Investigating instability of rumination and negative emotion. Journal of Clinical Psychology, 69, 1213-1227.

Selby, E.A., \& Joiner, T.E. (2009). Cascades of emotion: The emergence of Borderline Personality Disorder from emotional and behavioural dysregulation. Review of General Psychology, 13, 219-229.

Selby, E.A., Yen, S., \& Spirito, A. (2013). Time varying prediction of thoughts of death and suicidal ideation in adolescents: Weekly ratings over 6-month follow-up. Journal of Clinical Child and Adolescents Psychology, 42, 481-495.

Surrence, K., Miranda, R., Marroquín, B.M., \& Chan, S. (2009). Brooding and reflective rumination among suicide attempters: Cognitive vulnerability to suicidal ideation. Behaviour, Research and Therapy, 47, 803-808.

Swannell, S., Martin, G., Page, A., Hasking, P., \& St John, N. (2014). Prevalence of nonsuicidal self-injury (NSSI) in non-clinical samples; Systematic review, meta-analysis and meta-regression. Suicide and Life-Threatening Behavior, 44, 273-303.

Taliaferro, L.A., \& Muehlenkamp, J.J. (2015). Factors associated with current versus lifetime self-injury among high school and college students. Suicide and Life-Threatening Behavior, 45, 84-97.

Trompetter, H.R., de Kleine, E., \& Bohlmeijer, E.T. (2017). Why does positive mental health buffer against psychopathology? An exploratory study on self-compassion as a 
resilience mechanism and adaptive emotion regulation strategy. Cognitive Therapy $\&$ Research, 41, 459-468.

Turner, B.J., Yiu, A., Muehlenkamp, J.J., \& Chapman, A.L. (2016). Occurrence and cooccurrence of nonsuicidal self-injury and disordered eating in a daily diary study: Which behaviour, when? Psychiatry Research, 246, 39-47.

van den Brink, E., \& Koster, F. (2015). Mindfulness-based compassionate living. London/New York: Routledge.

Watkins, E., Scott, J., Wingrove, J., Rimes, K., Bathurst, N., Steiner, H., Kennell-Webb, S., Moulds, M., \& Malliaris, Y. (2007). Rumination-focused cognitive behaviour therapy for residual depression: A case series. Behaviour Research and Therapy, 45, 2144-2154.

Watkins, E.R. (2015). Rumination. In. R.A. Scott \& M.C. Buchmann (Eds). Emerging Trends in the Social and Behavioural Sciences. New York: John Wiley \& Sons.

Watson, D., Clarke, L.A., \& Tellegan, A. (1988). Development and validation of brief measures of positive and negative affect: The PANAS scales. Journal of Personality \& Social Psychology, 54, 1063-1070.

Whitlock, J., Muehlenkamp, J., Eckenrode, J., Purington, A., Abrams, G.B., Barriera, P., \& Kress, V. (2013). Nonsuicidal self-injury as a gateway to suicide in young adults. Journal of Adoelscent Health, 52, 486-192.

Xavier, A., Pinto-Gouveia, J.P., \& Cunha, M. (2016). Non-suicidal self-injury in adolescence: The role of shame, self-criticism and fear of self-compassion. Child Youth Care Forum, 45, 571-586. 
Figure 1. Final model showing standardised coefficients in predicting lifetime history of NSSI and suicide ideation. NSSI $=$ non-suicidal self-injury. $* * p<.01 * * * p<.001$

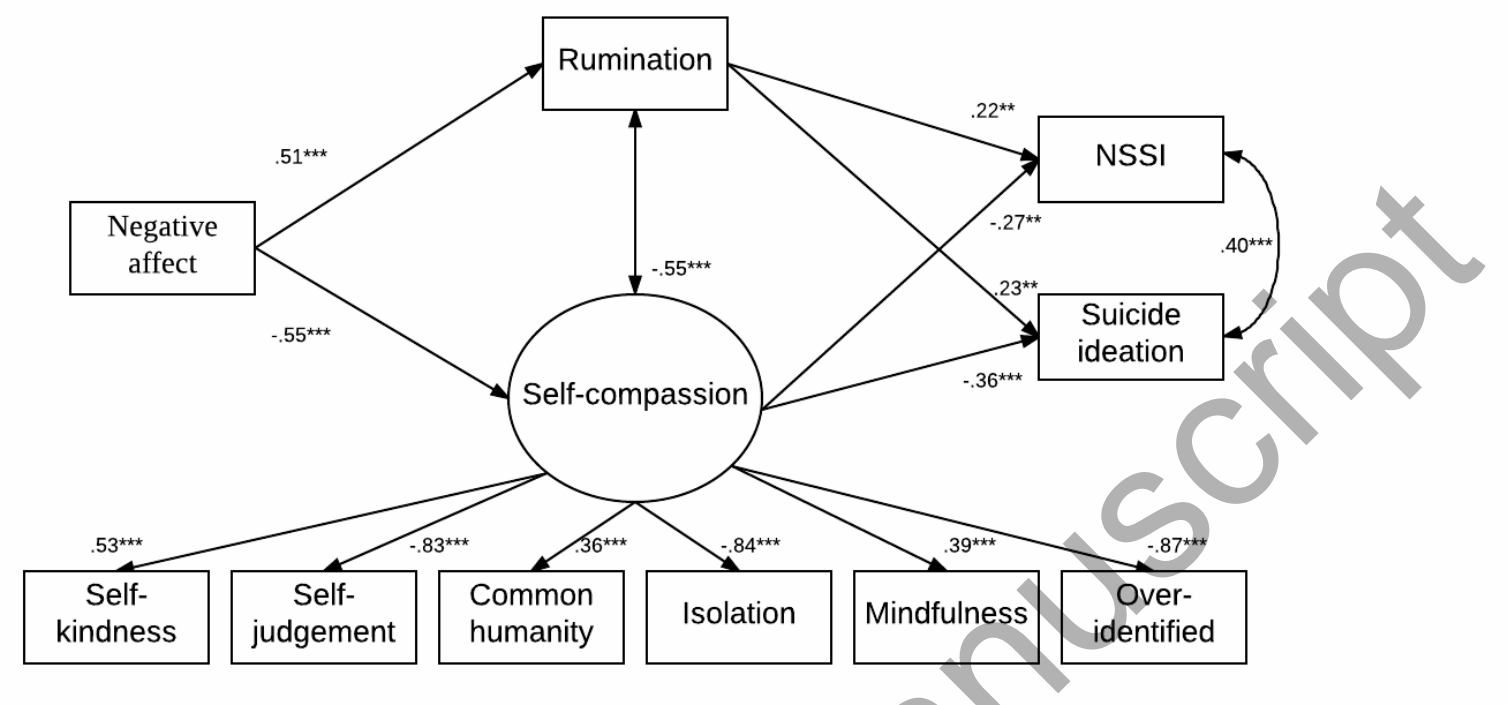


Table 1. Descriptive statistics and correlations between variables of interest

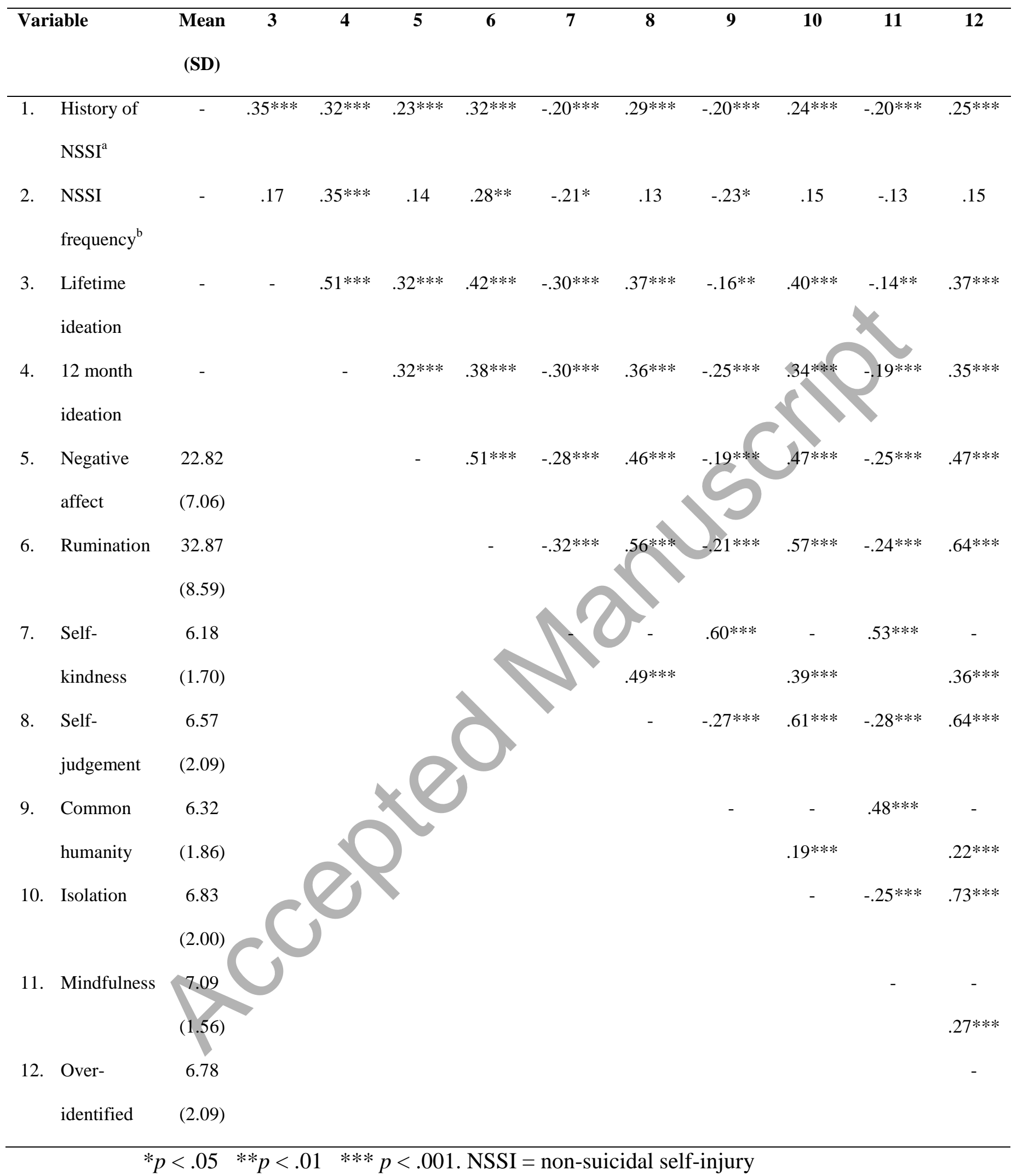

apoint biserial correlations for dichotomous variables; ${ }^{b}$ among people with a history of NSSI 
Table 2. Unstandardized coefficients for direct and indirect effects

Path

Coefficient Standard

p

$95 \% \mathrm{CI}$

error

\section{Lifetime NSSI and suicide ideation}

Direct effects

$\mathrm{NA} \rightarrow$ NSSI
$\begin{aligned} & \text { Rumination } \rightarrow \text { NSSI } \\ & \text { Self-compassion } \rightarrow \text { NSSI }\end{aligned}$
$\mathrm{NA} \rightarrow$ Suicide ideation

\section{2 month NSSI frequency and 12 month ideation}

Direct effects

$\begin{array}{lllll}\mathrm{NA} \rightarrow \mathrm{NSSI} & -.01 & .02 & .74 & -.04 \text { to } .03 \\ \text { Rumination } \rightarrow \text { NSSI } & .03 & .02 & .14 & -.01 \text { to } .06 \\ \text { Self-compassion } \rightarrow \text { NSSI } & -.55 & .21 & .009 & -.88 \text { to } .22\end{array}$




\begin{tabular}{|c|c|c|c|c|}
\hline NA $\rightarrow$ Suicide ideation & .01 & .02 & .61 & -.04 to .03 \\
\hline Rumination $\rightarrow$ Suicide ideation & .00 & .02 & .89 & -.03 to .04 \\
\hline Self-compassion $\rightarrow$ Suicide ideation & -.89 & .28 & .002 & -.1 .53 to -.11 \\
\hline NA $\rightarrow$ Rumination & .41 & .09 & $<.001$ & .30 to .58 \\
\hline NA $\rightarrow$ Self-compassion & -.05 & .01 & $<.001$ & -.08 to -.02 \\
\hline \multicolumn{5}{|l|}{ rect effects } \\
\hline $\mathrm{NA} \rightarrow \mathrm{NSSI}$ & .03 & .01 & .007 & .01 to .05 \\
\hline NA $\rightarrow$ Self-compassion $\rightarrow$ NSSI & .03 & .01 & .007 & 0.05 \\
\hline NA $\rightarrow$ Suicide ideation & .05 & .02 & & to .08 \\
\hline NA $\rightarrow$ Self-compassion $\rightarrow$ Suicide ideation & .05 & .02 & & .02 to .08 \\
\hline
\end{tabular}

Note. $\mathrm{NA}=$ negative affect, $\mathrm{NSSI}=$ non-suicidal self-injury 